\title{
Boris Induced Ventricular Tachycardia
}

\author{
Boos CJ*1,2,3, Girija A1 and Like $\mathbf{J}^{1}$ \\ ${ }^{1}$ Department of Cardiology, Poole Hospital NHS Foundation trust, UK \\ ${ }^{2}$ Dept of Postgraduate Medical Education, Bournemouth University, UK \\ ${ }^{3}$ Research Institute for Sport, Physical Activity and Leisure, Leeds Beckett University, Leeds, UK
}

*Corresponding author: Christopher J Boos, Department of Cardiology, Poole Hospital NHS Foundation Trust, Longfleet Rd. Poole, UK.
Received Date: November 08, 2019

Published Date: November 26, 2019

\begin{abstract}
A 93-year-old man with a known history of heart failure and a biventricular pacemaker inserted in 2012 presented with symptomatic palpitations and pre-syncope whilst watching Boris Johnson debating on live TV. He had known heart failure and biventricular pacemaker which had been inserted in 2011. His ECG confirmed ventricular tachycardia which was successfully cardioverted to sinus rhythm following an emergency cardioversion. He made an unremarkable recovery and was treated with increased Beta blockers and advised 'less Boris'.
\end{abstract}

\section{Case Report}

Mr W was a 93-year-old man admitted to Hospital with a sudden acute onset of symptomatic palpitations. He had been watching Boris Johnson stating his case to be prime minister on TV. He described becoming increasingly worked up by the impact of the debate just prior to the onset of his symptoms. His palpitations were described as being fast and regular and associated with shortness of breath, significant fatigue and light headedness. He had a history of symptomatic heart failure with broad left bundle branch and ischemic cardiomyopathy with an ejection fraction of $25 \%$ culminating in cardiac resynchronization with a pacemaker (CRTP) in 2011. He underwent an elective box change in 2018 and had been clinical well with NYHA III heart failure symptoms.

Figure 1: VT Case Report. 
On examination in Accident and Emergency $\mathrm{Mr} \mathrm{W}$ was obviously pale and breathless. He had a heart rate of 180 beats per minute and a palpable but week peripheral pulse. His blood pressure was $80 / 58 \mathrm{mmHg}$ and his heart sounds were normal. His respiratory rate was 28 breaths per minute and there were bilateral lower zone crepitations on auscultation. A 12 lead ECG confirmed the presence of a broad complex tachycardia consistent with fast ventricular tachycardia (Figure 1). He underwent rapid sequence induction of Anaesthesia and was successfully cardioverted to sinus rhythm with biventricular pacing capture (Figure 2) following a single 150J biphasic direct-current anterior posterior shock. 'It was either Boris or my wife's cooking. Having been married for more than 60 years without problems, would lead me to deduce it was Boris' he said. Mr W made an uneventful recovery. His beta blocker, which had been stopped by his GP in the last three years, was recommenced and was advised to avoid watching Boris. He was discharged to his own home within 48 hours of his admission.

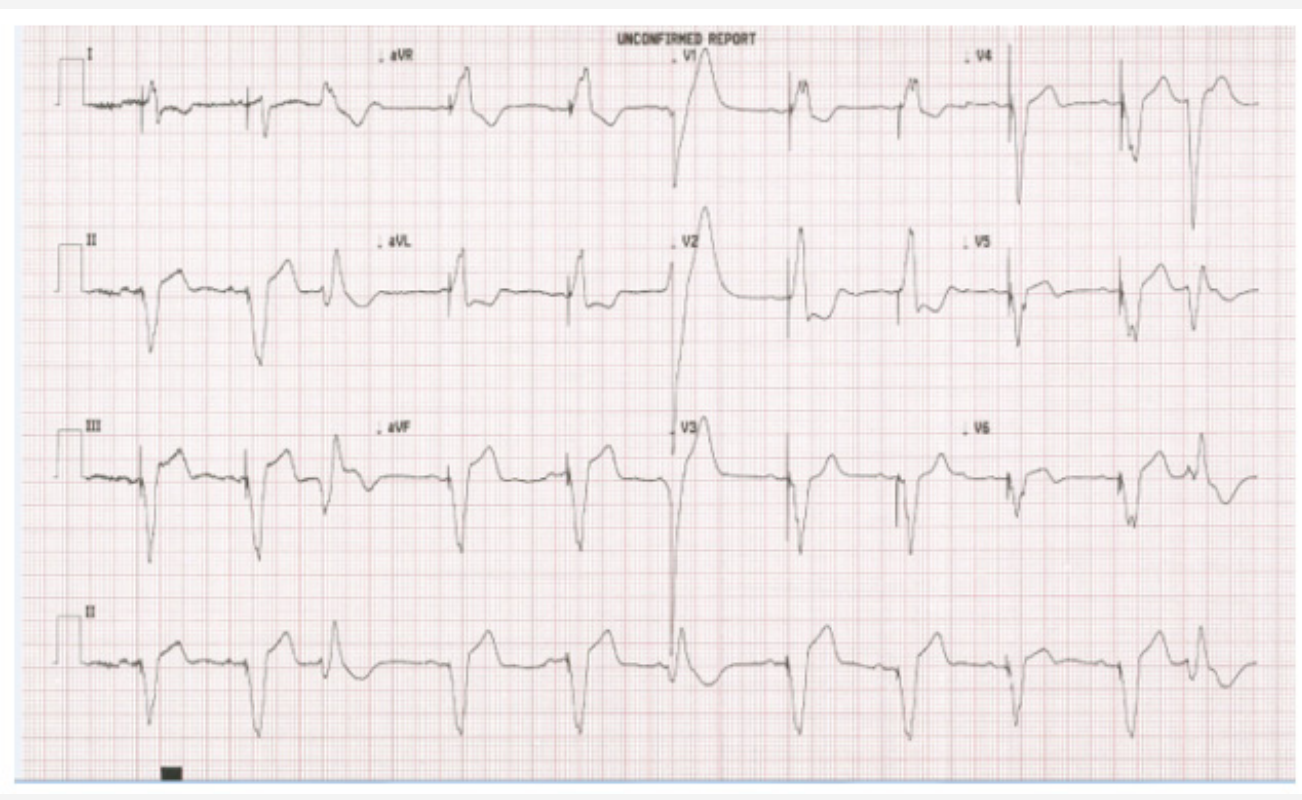

Figure 2: VT Case Report.

This case is a timely reminder of the fact that the choice of prime ministers does influence of the health of the nation beyond that of policy.

\section{Acknowledgement}

None.

\section{Conflict of Interest}

No conflict of interest. 